PROCEEDINGS OF THE

AMERICAN MATHEMATICAL SOCIETY

Volume 130, Number 6, Pages 1867-1873

S 0002-9939(01)06266-9

Article electronically published on November 15, 2001

\title{
COVERING MAPS THAT ARE NOT COMPOSITIONS OF COVERING MAPS OF LESSER ORDER
}

\author{
JERZY KRZEMPEK
}

(Communicated by Alan Dow)

\begin{abstract}
In $1995 \mathrm{~J} . \mathrm{W}$. Heath asked which exactly $n$-to-one maps are compositions of exactly $k$-to-one maps with $1<k<n$. This paper deals with compositions of covering maps. Exactly $n$-to-one covering maps on locally arcwise connected continua that are not factorable into covering maps of order $\leq n-1$ are constructed for all $n$ 's, and characterized in algebraic terms (fundamental groups). They are not proper compositions of exactly $k$-to-one maps, open maps, or locally one-to-one maps.
\end{abstract}

A (continuous) map is said to be of order $\leq n$ when each of its point-inverses has at most $n$ points. Maps of order $\leq 2$ are called simple.

Let us recall the following three problems from the literature each of them concerns maps between compact metric spaces.

Problem A (K. Borsuk, R. Molski [2]). Is every locally one-to-one map a composition of a finite number of simple locally one-to-one maps?1

Problem B (J.D. Baildon 1]). Is every open map of order $\leq n$ defined on a finitedimensional space necessarily a finite composition of simple open maps?

Problem C (J.W. Heath [6]). Under what circumstances are exactly $n$-to-one maps finite compositions of maps of lesser order 2

J. Dydak [3] showed that if $p$ is prime, then the map $z \mapsto z^{p}$ of the unit complex circle is not factorable into locally one-to-one maps of order $\leq p-1$. These same maps $z \mapsto z^{p}$ are not compositions of simple open maps (Baildon [1]). On the other hand, both Problems A and B have affirmative answers in the case of maps from zero-dimensional space 3 . Problem $\mathrm{C}$ was considered by L.R. Griffus [4. Lastly, let us mention that exactly four-to-one maps from the interval $[0,1]$ are not factorable

Received by the editors November 6, 2000 and, in revised form, January 9, 2001.

2000 Mathematics Subject Classification. Primary 54C10; Secondary 05C25.

Key words and phrases. Composition, covering map, locally one-to-one, open, exactly $k$-to-one map, group action, fundamental group.

${ }^{1} \mathrm{~A}$ map $f$ is locally one-to-one if each point in its domain has a neighborhood $U$ such that the restriction $f \mid U$ is one-to-one. Such maps were called elementary in [2, 3].

Borsuk and Molski 2 also asked another question: Does there exist a map of order $\leq n$ which is not a finite composition of simple maps? K. Sieklucki [10] proved that every map of order $\leq n$ defined on a finite-dimensional compact metric space is such a composition, and constructed an infinite-dimensional counter-example. For recent results on this problem see Krzempek 7.

${ }^{2} \mathrm{~A}$ function is called exactly $n$-to-one if each of its point-inverses consists of $n$ points.

${ }^{3}$ More precisely: Every clopen map of order $\leq n$ defined on a zero-dimensional metric space is a composition of $n-1$ simple clopen maps whose domains are metrizable (cf. Krzempek 7], 
into exactly two-to-one functions with finitely many discontinuities because there exist no finitely discontinuous exactly two-to-one functions from [0,1] (Heath [5]).

In this paper we show that for each $n$ there exists a connected finite graph $K$ with an exactly $n$-to-one covering map $f: K \rightarrow L$ that is not a composition of locally one-to-one maps of lesser order, open maps of lesser order, or exactly $k$-to-one maps of lesser order. Further, given a covering map, we represent its factorizations into covering maps by subgroup chains in the fundamental group of its image. As a result we characterize those infactorable covering maps in algebraic terms. We also find such maps among factors of the orbit maps induced by fixed-point-free actions of alternating groups $A(n)$.

A composition $r \circ p$, where $p$ is a map from a space $X$, is called proper if neither $p$ nor $r \mid p(X)$ is one-to-one. A covering map from a continuum is called prime if it is not one-to-one and is not a proper composition of covering maps. When we speak about a composition of exactly $k$-to-one maps $p$ and $r$, we do not mean that the $k$ 's for $p$ and $r$ are necessarily equal.

All spaces are meant to be Hausdorff and compact.

\section{Prime covering maps as examples to Problems A-C}

We shall frequently use the fact that every covering map onto a continuum is exactly $k$-to-one for some $k$. We start with an auxiliary example.

Example 1. Let $C_{1}$ be the unit complex circle $|z|=1$, and $C_{2}$ the circle $|z|=2$. Define $g_{n}: C_{1} \cup C_{2} \rightarrow C_{1}$ so that $g_{n} \mid C_{1}$ is the map $z \mapsto z^{n-1}$ and $g_{n} \mid C_{2}$ is $z \mapsto z / 2$. So, $g_{n}$ is an exactly $n$-to-one covering map. It is not a proper composition of exactly $k$-to-one covering maps. Indeed, suppose $g_{n}=r \circ p$, where $p$ is an exactly $k$-to-one covering map. Then $p \mid C_{1}$ is a covering map, and hence is exactly $k^{\prime}$-to-one for some $k^{\prime}$. If $r \mid p\left(C_{1} \cup C_{2}\right)$ is not one-to-one, then $k=k^{\prime}$ and it follows that $k=1$.

In order to obtain examples of exactly $n$-to-one prime covering maps with composite $n$ 's, we shall embed the domain of the maps $g_{n}$ defined above in a continuum.

Example 2. Consider three simple closed curves $C_{1}, C_{2}$, and $C_{3}$ joint as in Figure 1: $C_{1}$ is built of arcs $b_{1}, \ldots, b_{n-1}, C_{2}$ equals $b_{n}$, and $C_{3}$ is built of $a_{1}, \ldots, a_{n}$. Let $f_{n}$ be a map that takes $a_{1}, \ldots, a_{n}$ one along the other so that the arrows agree, and carries them onto $a$, and then takes $b_{1}, \ldots, b_{n}$ in the same manner, and carries them onto $b$. It is seen in the picture that $f_{n}$ is a covering map. It is not a proper composition of covering maps, for its restriction to $C_{1} \cup C_{2}$ is $g_{n}$ from Example 1

The reduction of Problems $\mathrm{A}-\mathrm{C}$ to the study of compositions of covering maps is done by means of two simple lemmata. The first is known (J. Mioduszewski [8]):

Lemma 1. Every exactly $k$-to-one locally one-to-one map is a covering map.

Proof. Suppose $f: X \rightarrow Y$ is exactly $k$-to-one and locally one-to-one. Take $y \in Y$ and pairwise disjoint open neighborhoods $U_{1}, \ldots, U_{k}$ of points $x_{1}, \ldots, x_{k} \in f^{-1}(y)$. The set $V=Y \backslash f\left[X \backslash\left(U_{1} \cup \ldots \cup U_{k}\right)\right] \ni y$ is open. If $f\left|U_{1}, \ldots, f\right| U_{k}$ are one-to-one, then $f^{-1}(V) \cap U_{1}, \ldots, f^{-1}(V) \cap U_{k}$ are carried onto $V$ (as $f$ is exactly $k$-to-one).

Theorem 1.1). There is no difficulty in showing that every locally one-to-one map of order $\leq 2^{n}$ between zero-dimensional compact spaces is a composition of $n$ simple locally one-to-one maps. 


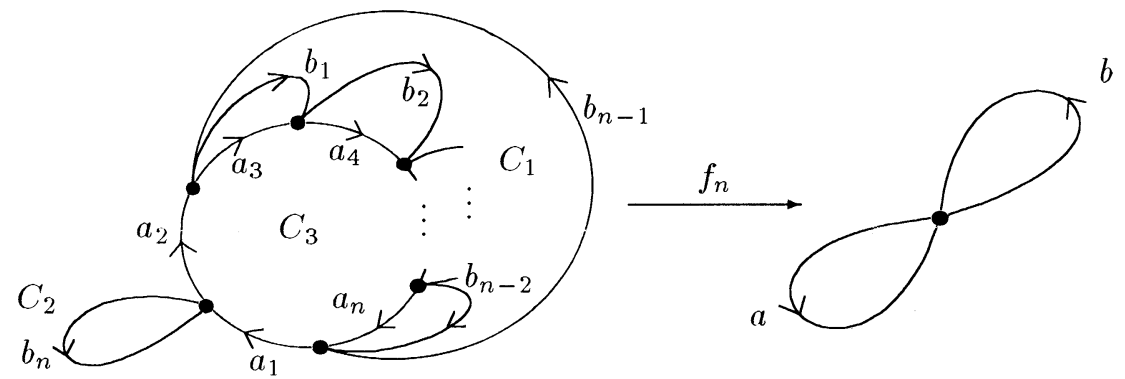

FIGURE 1.

Lemma 2. Let $r \circ p$ be a local homeomorphism 4 where $p$ is a map onto the domain of $r$. Then the statements (a)-(c) below are equivalent, and they follow from (d).

(a) $p$ and $r$ are local homeomorphisms.

(b) $p$ and $r$ are locally one-to-one.

(c) $p$ and $r$ are open.

(d) $p$ is exactly $k$-to-one.

Proof. The implications $(\mathrm{a}) \Rightarrow(\mathrm{b}),(\mathrm{a}) \Rightarrow(\mathrm{c})$, and $[(\mathrm{b}) \wedge(\mathrm{c})] \Rightarrow(\mathrm{a})$ are obvious. Independent of the statements (a)-(d), $p$ is locally one-to-one and $r$ is open. Therefore, Lemma 1 yields the implication $(\mathrm{d}) \Rightarrow(\mathrm{c})$. It remains to show that $p$ is open iff $r$ is locally one-to-one. Write $f=r \circ p, p: X \rightarrow Y$ and $r: Y \rightarrow Z$, where $p(X)=Y$.

Suppose $p$ is open. Take $y \in Y, x \in p^{-1}(y)$, and an open set $U \ni x$ such that $f \mid U$ is one-to-one. Then $p(U)$ is an open neighborhood of $y$, and $r \mid p(U)$ is one-to-one.

Suppose $r$ is locally one-to-one. For each $x \in X$ take an open set $V \ni p(x)$ such that $r \mid V$ is one-to-one. If $U \ni x$ is open, and $p(U) \subset V$, then $p(U)=V \cap r^{-1} f(U)$ is open. Thus, $X$ has a base that consists of sets $U$ whose images $p(U)$ are open.

We assume that spaces are Hausdorff and compact. In consequence a map is a covering map iff it is a local homeomorphism. This is a corollary to Lemma 2.

Theorem 1. Suppose that $f$ is a covering map from a Hausdorff continuum. Then the following statements are equivalent:

(a) $f$ is a proper composition of covering maps.

(b) $f$ is a proper composition of locally one-to-one maps.

(c) $f$ is a proper composition of open maps.

(d) $f$ is a proper composition of exactly $k$-to-one maps.

It follows that the exactly $n$-to-one prime covering maps $f_{n}$ defined in Example 2 are not proper compositions of locally one-to-one maps, open maps, or exactly $k$-toone maps. Neither are the maps $z \mapsto z^{p}$ of the unit complex circle where $p$ are prime (cf. Baildon [1], Dydak [3]). Finally, by the implication (d) $\Rightarrow($ a) of Lemma 2 the maps $g_{n}$ from Example 1 are not proper compositions of exactly $k$-to-one maps.

\section{Group-theoretic approach to COMPositions of COVERING Maps}

Here is some terminology associated with group actions where $X$ is a space and $G$ is a finite group. When some homeomorphisms of $X$ form a group isomorphic to $G$, we say that $G$ acts on the space $X$. We identify each element of $G$ with a

\footnotetext{
${ }^{4}$ Open locally one-to-one maps are called local homeomorphisms.
} 
respective homeomorphism. When all these homeomorphisms except the identity are fixed-point-free, the group action is also called fixed-point-free. We write $X / G$ for the orbit space with the quotient topology, and $q_{G}: X \rightarrow X / G$ for the orbit map that assigns to each point $x \in X$ its $G$-orbit $\{g(x): g \in G\}$. We say that this map is induced by the group action. It is readily seen that the orbit space is Hausdorff, and the orbit map is open. Moreover, the orbit map is a covering map if the action is fixed-point-free.

If $H \leq G$ is a normal subgroup (we write $H \triangleleft G$ ), then each coset $H \circ g$ corresponds to a homeomorphism $X / H \rightarrow X / H$ that carries the $H$-orbit of any $x \in X$ into the $H$-orbit of $g(x)$. It is easy to check that this correspondence is a group isomorphism, a $G / H$-action on the orbit space $X / H$. As a result we obtain

Proposition. Consider an action of a finite group $G$ (on any space). Then each subgroup $H \leq G$ induces a factorization $r \circ q_{H}=q_{G}$ of the orbit map $q_{G}$ into open maps $q_{H}$ and $r$. If $H \triangleleft G$, then $r$ is induced by a $G / H$-action.

The first corollary is that if a prime covering map is induced by a fixed-point-free group action, then the underlying group is $\mathbb{Z}_{p}$ with some prime number $p$. Secondly, each $2^{n}$ element group $(G, \cdot, 1)$ contains subgroups $\{1\}=H_{0} \leq H_{1} \leq \ldots \leq H_{n}=G$, where $H_{k}$ has $2^{k}$ elements for $k=0,1, \ldots, n$; see statement 5.3.1(ii) in D.J.S. Robinson [9]. Therefore, the orbit map induced by an action of any $2^{n}$ element group is a composition of $n$ simple open maps (cf. Baildon [1], Corollary 6).

We write $\left(X, x_{0}\right)$ for a space $X$ with a point $x_{0} \in X, \pi\left(X, x_{0}\right)$ for the fundamental group of $\left(X, x_{0}\right), f:\left(X, x_{0}\right) \rightarrow\left(Y, y_{0}\right)$ for a map $f: X \rightarrow Y$ such that $f\left(x_{0}\right)=y_{0}$, and $f_{\#}: \pi\left(X, x_{0}\right) \rightarrow \pi\left(Y, y_{0}\right)$ for the homomorphism that carries any homotopy class $[\omega] \in \pi\left(X, x_{0}\right)$ of loops into the class $[f \circ \omega]$. Recall that if $f$ is a covering map, then the homomorphism $f_{\#}$ is a monomorphism (cf. E. Spanier [11], Theorem 2.3.4).

Theorem 2. Suppose $X$ is a locally arcwise connected Hausdorff continuum, and suppose $f:\left(X, x_{0}\right) \stackrel{\text { onto }}{\longrightarrow}\left(Y, y_{0}\right)$ is a covering map. Consider all factorizations

$$
\left(X, x_{0}\right)=\left(X_{0}, x_{0}\right) \stackrel{p_{1}}{\rightarrow}\left(X_{1}, x_{1}\right) \stackrel{p_{2}}{\rightarrow} \ldots \stackrel{p_{n}}{\rightarrow}\left(X_{n}, x_{n}\right)=\left(Y, y_{0}\right)
$$

of $f$ into surjective covering maps $p_{1}, \ldots, p_{n}$, and all subgroup chains

$$
f_{\#} \pi\left(X, x_{0}\right)=H_{0} \leq H_{1} \leq \ldots \leq H_{n}=\pi\left(Y, y_{0}\right) .
$$

Then the formulae $H_{k-1}=\left(p_{n} \circ \ldots \circ p_{k}\right)_{\#} \pi\left(X_{k-1}, x_{k-1}\right)$, where $k=1, \ldots, n$, define a bijective correspondence between these factorizations and subgroup chains. The order of each map $p_{k}$ equals the index of the group $H_{k-1}$ in $H_{k}$. A map $p_{k}$ is induced by a fixed-point-free group action on $X_{k-1}$ iff $H_{k-1} \triangleleft H_{k}$.

Proof. For $n=1$ the chain $f_{\#} \pi\left(X, x_{0}\right) \leq \pi\left(Y, y_{0}\right)$ corresponds to $f$. The equality and equivalence directly follow from Theorems 2.3.9, 2.3.12 and Corollary 2.6.3 in [11].

Let $n=2$. From Lemma 2.5.11 and Theorem 2.5.13 in [11] we infer that the formula $H_{1}=p_{2 \#} \pi\left(X_{1}, x_{1}\right)$ defines a bijective correspondence between groups $H_{1}$ such that $f_{\#} \pi\left(X, x_{0}\right) \leq H_{1} \leq \pi\left(Y, y_{0}\right)$ and covering maps $p_{2}:\left(X_{1}, x_{1}\right) \stackrel{\text { onto }}{\longrightarrow}\left(Y, y_{0}\right)$ such that $f_{\#} \pi\left(X, x_{0}\right) \leq p_{2 \#} \pi\left(X_{1}, x_{1}\right) \leq \pi\left(Y, y_{0}\right)$. For each $p_{2}$ define $p_{1}:\left(X, x_{0}\right) \rightarrow\left(X_{1}, x_{1}\right)$ as the unique lifting, i.e. a map such that $f=p_{2} \circ p_{1}$ (see Theorems 2.4.5 and 2.2.2 in [11]). This $p_{1}$ is a covering map by Lemma 2.5.1 in [11]. The needed equalities and equivalences result from the case $n=1$ and the fact that the monomorphism $p_{2 \#}$ carries the groups $p_{1 \#} \pi\left(X, x_{0}\right)$ and $\pi\left(X_{1}, x_{1}\right)$ onto $f_{\#} \pi\left(X, x_{0}\right)$ and $H_{1}$ respectively.

The easy induction step is left to the reader. 
According to Theorem 10 the following corollary answers the question "Under what circumstances..." in Problem $\mathrm{C}$ for covering maps defined on locally arcwise connected Hausdorff continua.

Corollary. A covering map $f: X \stackrel{\text { onto }}{\longrightarrow} Y$ from a locally arcwise connected Hausdorff continuum $X$ is prime iff the subgroup $f_{\#} \pi\left(X, x_{0}\right)$ is maxima 5 in $\pi\left(Y, f\left(x_{0}\right)\right)$.

Theorem 3. Suppose $(G, \cdot, 1)$ is a finite group that acts without fixed points on a locally arcwise connected Hausdorff continuum. Consider all factorizations

$$
q_{G}=p_{n} \circ \ldots \circ p_{1}
$$

of the orbit map $q_{G}$ into surjective covering maps $p_{1}, \ldots, p_{n}$, and all subgroup chains

$$
\{1\}=H_{0} \leq H_{1} \leq \ldots \leq H_{n}=G
$$

Then between these factorizations and chains there is a bijective corresponedence such that for each $k=1,2, \ldots, n$ the index of the group $H_{k-1}$ in $H_{k}$ equals the order of $p_{k}$, and $H_{k-1} \triangleleft H_{k}$ iff $p_{k}$ is induced by a fixed-point-free group action.

Proof. Write $q_{G}:\left(X, x_{0}\right) \stackrel{\text { onto }}{\longrightarrow}\left(Y, y_{0}\right)$. Let $\iota: \pi\left(Y, y_{0}\right) \rightarrow \pi\left(Y, y_{0}\right) / q_{G \#} \pi\left(X, x_{0}\right)$ denote the canonical homomorphism that carries each homotopy class $[\omega] \in \pi\left(Y, y_{0}\right)$ into the coset of $q_{G \#} \pi\left(X, x_{0}\right)$ containing this class. By Corollary 2.6.3 in [1] there is an isomorphism $\psi: \pi\left(Y, y_{0}\right) / q_{G \#} \pi\left(X, x_{0}\right) \rightarrow G$. If $q_{G \#} \pi\left(X, x_{0}\right) \leq H \leq H^{\prime} \leq \pi\left(Y, y_{0}\right)$, then the index of $H$ in $H^{\prime}$ equals the index of $\psi[\iota(H)]$ in $\psi\left[\iota\left(H^{\prime}\right)\right]$, and $H \triangleleft H^{\prime}$ iff $\psi[\iota(H)] \triangleleft \psi\left[\iota\left(H^{\prime}\right)\right]$. The use of Theorem 2 completes the proof.

The foregoing theorem shows that all factorizations of "sufficiently good" orbit maps were described in our Proposition. This enables another construction of prime covering maps with composite orders.

Example 3. Consider $A(4)$, the group of even permutations of a four point set. It is well-known that every finite group acts without fixed points on a connected finite graph. Take such an $A(4)$-action on a graph $K$. Consider all paths in Figure 2 from the trivial subgroup to the whole of $A(4)$, and all factorizations of the orbit map $q_{A(4)}$ into prime covering maps. Theorem 3 establishes an "accurate" correspondence between these paths and factorizations. The line that joins a three element subgroup, say $H$, with the whole of $A(4)$ corresponds to a four-fold covering map $K / H \rightarrow K / A(4)$. It is prime as $A(4)$ contains no six element subgroup.

There is a classical theorem on permutation groups that for each $n>4$ the alternating group $A(n)$ does not contain subgroups of indices $2,3, \ldots, n-1$. It results from statements 1.6.9 and 3.2.16 in [9], and enables us to construct exactly $n$-to-one prime covering maps for $n>4$ as we did in Example 3 for $n=4$. We need to see that if $A(n)$ denotes the group of even permutations of the set $\{1, \ldots, n\}$, then the subgroup $H$ of all $\sigma \in A(n)$ such that $\sigma(1)=1$ has index $n$ and is maximal in $A(n)$.

\footnotetext{
${ }^{5} \mathrm{~A}$ proper subgroup is called maximal if it is not contained in any larger proper subgroup.

${ }^{6}$ One more fact is needed: The core $G_{A(n)}$ of any subgroup $G$ in $A(n)$ is a normal subgroupthis is apparent in the definition of core; see [9], p. 16.
} 


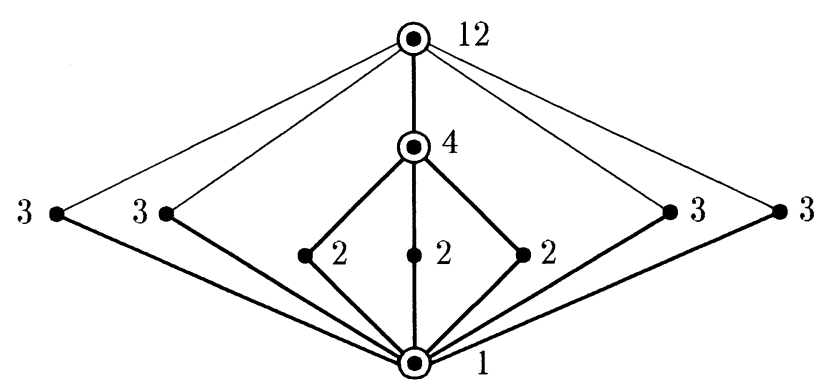

Figure 2. The subgroup lattice of the alternating group $A(4)$ (cf. [9, the beginning of the proof of 3.2.1, and Exercise 1.3.3). Subgroups [ones normal in $A(4)$ ] are denoted by [framed] points with the numbers of elements. Inclusions are denoted by lines, thick when a subgroup is normal in the next larger one.

\section{REMARKS}

We infer from Theorem 3 that the orbit map of a fixed-point-free group action on a "nice" space contains full information on the subgroup lattice of the underlying group. For example, it is easily checked that this group is soluble (see 9 for a definition and basic properties) iff its orbit map is a composition of prime covering maps induced by fixed-point-free group actions (then these groups are $\mathbb{Z}_{p}$ with prime $p$ 's).

In Example 3 we found an exactly four-to-one prime covering map among factors of an orbit map $q_{A(4)}$. On the other hand, it is seen in Figure 2 that factorizations of this $q_{A(4)}$ into prime covering maps have different lengths: 2 or 3 . Indeed, a group-theoretic result (see 10.3.5 in [9]) and our Theorem[3imply that:

For every covering map $f$ induced by a fixed-point-free group action on a locally arcwise connected Hausdorff continuum the following statements are equivalent:

(a) No factorization of $f$ into prime covering maps contains an exactly $n$-to-one map with composite $n$.

(b) All factorizations of $f$ into prime covering maps have the same length.

(c) This $f$ is factorable into prime covering maps $p_{1}, \ldots, p_{n}$ such that all compositions $p_{j} \circ \ldots \circ p_{i}$, where $j \geq i$, are induced by fixed-point-free group actions.

A map $f$ is called $n$-crisp if for each proper subcontinuum $C$ in the image there are exactly $n$ components of $f^{-1}(C)$, and $f$ maps each of these components homeomorphically onto $C$. Griffus [4] showed that every $n$-crisp map from a continuum is a covering map. His problem remains unsolved: Do there exist prime $n$-crisp maps from continua for composite n's?

\section{REFERENCES}

1. J.D. Baildon, Open simple maps and periodic homeomorphisms, Proc. Amer. Math. Soc. 39 (1973), 433-436. MR 48:2976

2. K. Borsuk, R. Molski, On a class of continuous mappings, Fund. Math. 45 (1957), 84-98. MR 21:858

3. J. Dydak, On elementary maps, Colloq. Math. 31 (1974), 67-69. MR 51:6696

4. L.R. Griffus, Exactly k-to-1 Maps Between Metric Continua, Ph.D. thesis, Auburn University, 1996. 
5. J.W. Heath, Every exactly 2-to-1 function on the reals has an infinite set of discontinuities, Proc. Amer. Math. Soc. 98 (1986), 369-373. MR 87i:54031

6. J.W. Heath, Exactly $k$-to-1 maps: from pathological functions with finitely many discontinuities to well-behaved covering maps, in: Continua. With the Houston Problem Book, 89-102, Lecture Notes in Pure and Appl. Math. 170, Dekker, New York, 1995. MR 96d:54015

7. J. Krzempek, Compositions of simple maps, Fund. Math. 162 (1999), 149-162. MR 2001b:54018

8. J. Mioduszewski, On two-to-one continuous functions, Dissertationes Math. (Rozprawy Mat.) 24 (1961). MR 26:3021

9. D.J.S. Robinson, A Course in the Theory of Groups, Springer, New York, 1996. MR 96f:20001

10. K. Sieklucki, On superposition of simple mappings, Fund. Math. 48 (1960), 217-228. MR 22:2981

11. E. Spanier, Algebraic Topology, Springer, New York, 1981. MR 83i:55001

Institute of Mathematics, Silesian Technical University, Kaszubska 23, PL-44-100 Gliwice, Poland

E-mail address: krzem@zeus.polsl.gliwice.pl 